\section{GOBERNANZA DE LA PLANIFICACIÓN TERRITORIAL Y EL RIESGO EN CHILE: EL CASO DE SAN BERNARDO Y CALERA DE TANGO}

Josefa Macarena Espinace Vidal ${ }^{{ }^{*}}$

\section{RESUMEN}

Se presenta un análisis y comparación respecto de la incorporación de la Gestión del Riesgo de Desastres en los instrumentos de ordenamiento y planificación territorial de las comunas de San Bernardo y Calera de Tango, en Santiago de Chile, identificando factores críticos que determinan las diferencias entre ellas en función de su nivel de riesgo. Si bien estas comunas peri-urbanas son colindantes y poseen características geográfico-físicas similares, cada una ha ido adoptando perfiles singulares de acuerdo a su funcionalidad dentro de la Región Metropolitana. Luego del análisis de los instrumentos de planificación territorial de cada comuna y de la información obtenida mediante entrevistas en cada municipio, fue posible identificar que la gestión local se encuentra limitada porque debe absorber las decisiones que desde los macro niveles territoriales se definen y que no siempre van en concordancia con la visión local. Ambas comunas han tenido que adecuarse a los cambios de uso de suelo que la región ha establecido según las necesidades que surgen desde el centro urbano de esta, lo que ha generado una tensión entre la visión local y regional, que se concreta en los instrumentos de planificación. Este escenario finalmente desfavorece la gestión de riesgo municipal.

\section{PALABRAS CLAVE}

Factores subyacentes del riesgo, Ordenamiento territorial, Planificación territorial, Gestión del riesgo de desastres, Gobernanza

\section{TERRITORIAL PLANNING AND RISK GOVERNANCE IN CHILE: THE CASE OF SAN BERNARDO AND CALERA DE TANGO}

\section{ABSTRACT}

A comparative analysis is presented regarding the integration of Disaster Risk Management on land use planning in the communes of San Bernardo and Calera de Tango in Santiago of Chile. This aims to differentiate critical factors between these two locations in relation to their exposure and risk levels. Although these peri-urban municipalities are adjacent to each other and share various similar geographic-physical characteristics, both have developed different urban features in compliance with distinct functionalities within the Metropolitan Region of Chile. Through the analysis of territorial planning instruments and primary data obtained by interviews, the study points out that local managements are limited because they must 'absorb' the decisions that are defined from macro territorial levels, which not always correspond to local aspirations and visions. Both communes have had no choice but to accept land use changes that the urban center region has established according to its needs. This has generated a tension between the local and regional development visions, which is manifested in different planning instruments. Ultimately, this scenario creates disadvantages for municipal risk management.

\section{KEYWORDS}

Underlying factors of risk, Territorial ordering, Territorial planning, Disaster risk management, Governance

\author{
1. Departamento de \\ Geografía, Universidad de \\ Chile, Santiago, Chile. \\ *Autor de correspondencia: \\ jo.espinace@gmail.com

\section{RECIBIDO} \\ 22 de marzo de 2020

\section{ACEPTADO} \\ 13 de mayo de 2020

\section{PUBLICADO} \\ 1 de julio de 2020

\section{Formato cita} \\ Recomendada (APA): \\ Espinace Vidal, J.M. \\ (2020). Gobernanza de la \\ planificación territorial y el \\ riesgo en Chile: El caso de \\ San Bernardo y Calera de \\ Tango. Revista de Estudios \\ Latinoamericanos sobre \\ Reducción del Riesgo de \\ Desastres REDER, 4(2), 7-21.
}

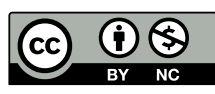

Todos los artículos publicados en REDER siguen una política de Acceso

Abierto y se respaldan en una Licencia CreativeCommons Atribución-NoComercial 4.0 Internacional.

Revista de Estudios Latinoamericanos sobre Reducción del Riesgo de Desastres (REDER)

Diseño: Lupe Bezzina Tipografía: Hospital 


\section{CONTEXTO GENERAL}

La ciudad de Santiago de Chile se encuentra expuesta a múltiples amenazas que en su historia han destruido sus construcciones y que ha tenido distintos niveles de afectación en la población y sus bienes. Hoy en día, bajo el contexto de un mundo globalizado, Santiago es uno de los centros urbanos que han adquirido un nuevo e importante rol en el sistema regional y global. Las ciudades son el escenario de la localización de sedes comerciales y financieras, que traen consigo una gama de servicios y equipamientos, estos a su vez, generan la centralización y concentración de población y funciones. La Región Metropolitana (RM), mayoritariamente de carácter urbano, alberga el $40,5 \%$ del total de la población a nivel país, de las cuales el $96,3 \%$ vive en zonas urbanas y el 3,7\% en zonas rurales (INE, 2018). Este escenario ha sido complejo para esta urbe, ya que no siempre da abasto con la gran cantidad de población que alberga, por lo cual se genera un crecimiento desregulado y poco planificado de la ciudad. Lo anterior, sumado al poder que la globalización y el modelo económico neoliberal les han otorgado a las decisiones individuales en la configuración del territorio, revela algunas de las causas fundamentales de la construcción de escenarios de riesgo de desastres (Sánchez, 2010).

Es posible afirmar entonces, que el ordenamiento territorial constituye un factor subyacente del riesgo, ya que condiciona el nivel de exposición y vulnerabilidad de la población ante una amenaza. Son las características territoriales previas a la generación de un evento disruptivo, las que favorecen o no el impacto negativo de estos, y que se relacionan con problemas causados por los procesos que se han generado producto de un inadecuado crecimiento urbano. En este sentido, un ordenamiento territorial eficaz que considere tanto las características físico-naturales como humanas y sociales de un espacio, podría considerarse una estrategia que previene la construcción de nuevos riesgos y ayuda a que no se agudicen aún más los ya existentes.

Hoy en día, los instrumentos de ordenamiento territorial a nivel comunal no consideran la Gestión del Riesgo de Desastres (GRD) como una herramienta válida para lograr un desarrollo sustentable, por otra parte, el marco normativo e institucional respecto de la reducción del riesgo de desastres no se constituye como un cuerpo de normas e instituciones articuladas entre sí, es más bien, un conjunto de acciones puntuales vinculadas a cier tos sectores de manera segmentada: no existiendo una normativa sistémica en torno a la temática (Barrenechea-Riveros, 2016). Pero este escenario aun cuando es complejo y limita el desarrollo de una GRD eficaz en los centros urbanos, es también una oportunidad para conformar asentamientos sustentables basados en una cultura de la prevención que inicie desde los niveles locales, sean estos individuales como comunales (Sánchez, 2010).

La presente investigación se basa en el estudio y análisis de dos comunas localizadas en la zona sur de la Región Metropolitana de Chile, las cuales son San Bernardo y Calera de Tango (Ver Figura 1). Se realiza una comparación entre ellas con el objetivo de identificar y establecer los factores críticos que influyen en los niveles de eficacia de la incorporación de la GRD en los Instrumentos de Planificación Territorial (IPTs). Esta área de estudio fue elegida luego de obtener los resultados de la Encuesta de Identificación de Factores Subyacentes del Riesgo que aplica la Oficina Nacional de Emergencia del Ministerio del Interior y Seguridad Pública (ONEMI). De este instrumento, cada comuna obtiene como resultado el Índice Comunal de Factores Subyacentes del Riesgo (ICFSR), el cual es de carácter cuantitativo y permite conocer el desempeño socioterritorial en torno a ciertas variables e identificar umbrales de riesgo que deberán luego ser interpretados y contextualizados conforme a la realidad comunal, y finalmente ser trabajados para superar aquéllos que se encuentran deficientes (ONEMI, 2017).

A nivel nacional, 60 comunas fueron encuestadas en 2017, de todas ellas la comuna mejor evaluada y que se encuentra en la RM fue San Bernardo con un ICFSR de un $12 \%$ lo que equivale a un nivel de riesgo bajo. Por otro lado, la comuna de Calera de Tango obtuvo un ICFSR de un $56 \%$ lo que equivale a un nivel de riesgo alto, siendo la que obtuvo el peor resultado dentro de la RM y la novena comuna con peor resultado a nivel nacional, es decir, existe una gran diferencia en los resultados de ambas comunas estudiadas.

Aunque son comunas colindantes, han desarrollado dinámicas y ocupación del territorio diferentes en torno a las funciones que les ha atribuido la región a través de los instrumentos de planificación. Sin embargo, la normativa no necesariamente ha respondido a las necesidades de cada comuna, por lo que comienzan a generarse tensiones en el uso del territorio, trayendo por 
consecuencia la creación de nuevos escenarios de riesgo. La gran diferencia de resultados entre ambas comunas se debe a que poseen características particulares, las cuales, al ser identificadas y comparadas, permiten establecer criterios y factores que mediante el ordenamiento y planificación territorial facilitan y/o condicionan los mecanismos establecidos para concretar una GRD con éxito.

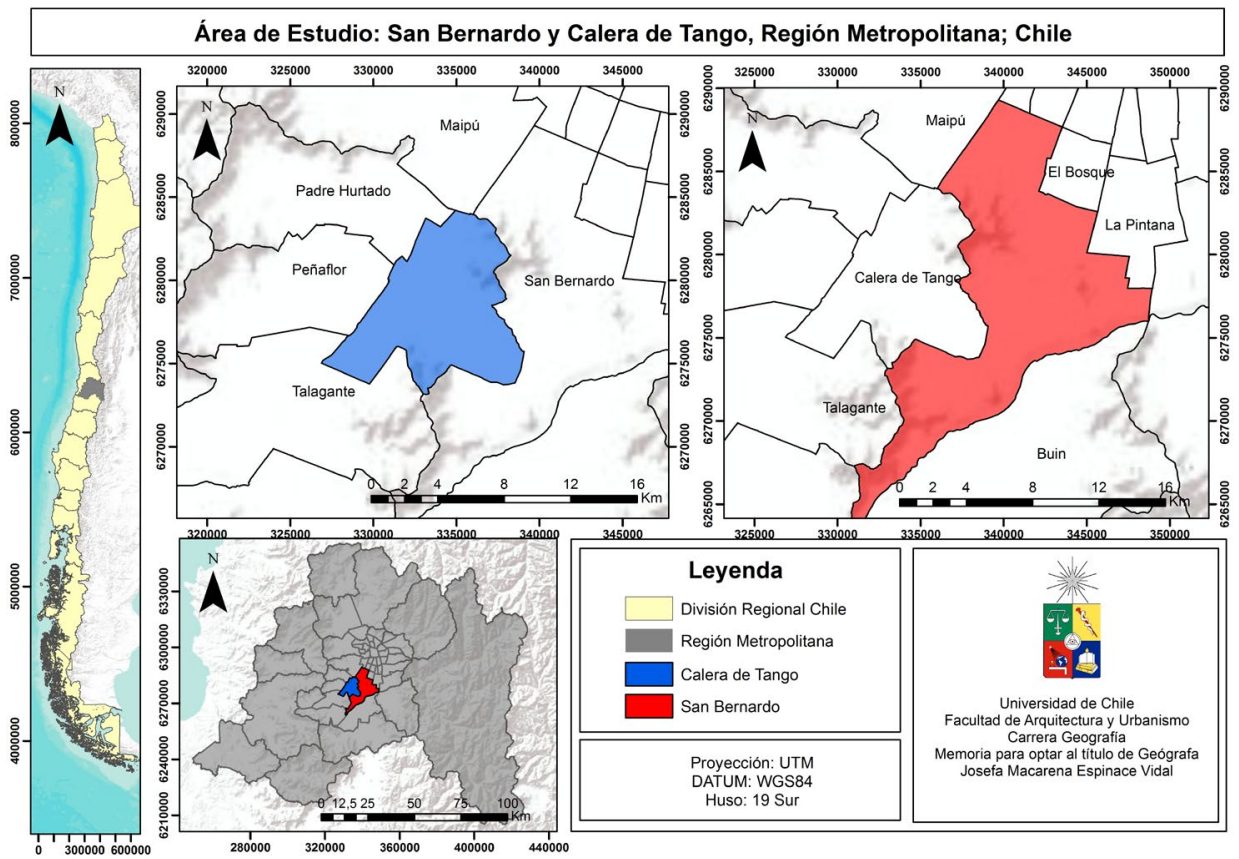

Figura 1. San Bernardo y Calera de Tango, Región Metropolitana, Chile Fuente: Autora, 2020

\section{MARCO TÉORICO}

\section{Ordenamiento y planificación territorial en un contexto de riesgo}

La configuración del territorio se va modelando dependiendo de los propósitos que emanan desde la sociedad y su organización, lo cual se logra principalmente a través del ordenamiento territorial, que implica, según Gross (1998), la búsqueda de la disposición correcta, equilibrada y armónica de la interacción de los componentes del territorio. En otras palabras, se concreta al identificar, distribuir, organizar y regular las actividades humanas en un espacio determinado, de acuerdo a ciertos criterios y prioridades que indican las proyecciones políticas económicas, sociales, culturales y ambientales de una sociedad (Gómez-Orea, 2007).

El ordenamiento territorial contiene los principios que se dan para ocupar el territorio. Estos lineamientos, para que se hagan efectivos, deben definir objetivos y luego aplicarlos a través de planes y estrategias, ámbito que le corresponde a la planificación territorial. Esta última se concreta a través de instrumentos que influyen tanto a nivel nacional como local, y que involucran a distintos actores. En la Ley General de Urbanismo y Construcciones de Chile (LGUC), se encuentran contenidas las funciones y objetivos de los instrumentos de planificación territorial, los cuales se ejercen a distintas escalas territoriales, ya sea nacional, regional, provincial y comunal.

Según Arenas et al. (2010, p.2), "la mayor parte de los espacios urbanos chilenos presenta situaciones de riesgo, debido a la ocupación no solo irregular y desprovista de planificación territorial, sino también a la presión ejercida desde el sector inmobiliario para la construcción en tales lugares"; lo que es facilitado por el deficiente control y normativa referente a la seguridad de la población y también respecto al uso y construcción del territorio, lo que finalmente genera el aumento de los factores de riesgo (Mansilla, 2000). Es por esto que la prevención de este tipo de escenarios está directamente relacionada con la imposición de restricciones en el uso del espacio que puede ejercer el ordenamiento y planificación territorial (Gómez Orea, 1994 en Ayala-Carcedo, 2001)

En el Plan Estratégico Nacional para la Gestión del Riesgo de Desastres 2015-2018 (2016, p.57), se da énfasis a que "es esencial reforzar la inclusión vinculante de la prevención de riesgos dentro de los instrumentos de planificación territorial identificando, por un lado, restricciones en las áreas críticas para los asentamientos humanos, infraestructura y patrimonio natural, y, por otro, 
delimitando aquellas áreas y ecosistemas que ameritan protegerse, resguardarse o restaurarse para reducir los riesgos o amortiguar los impactos". La manera de lograr este objetivo es a través del mejoramiento de la coordinación entre las instituciones responsables y participantes tanto del ámbito de la gestión del riesgo como del ordenamiento territorial, permitiendo generar finalmente planes y herramientas coherentes en las diferentes escalas del territorio que logren proteger el uso adecuado del suelo en función de los contextos locales, considerando las amenazas y vulnerabilidades presentes, como una forma de prevenir, mitigar y finalmente reducir el riesgo de desastres (Castro et al., 2013).

\section{Gobernanza en la Gestión del Riesgo de Desastres}

La GRD es un proceso permanente que, dentro de su complejidad, persigue diferentes objetivos. Dentro de un análisis más profundo, se considera que su fin es el trabajo conjunto de los diferentes elementos que conforman el territorio y de todos los niveles en los cuales se llevan a cabo trabajos intersectoriales, promoviendo la acción conjunta de todos los actores, provocando que la gestión sea mucho más efectiva en pos de reducir el riesgo de desastres. En este sentido, es posible identificar un proceso de descentralización, ya que, de esta manera las comunidades e inversiones locales pueden involucrarse y con esto es posible lograr resultados efectivos de intervención (Cardona, 2008 en Orellana, 2015), con lo que se da paso a la gestión local del riesgo (Orellana, 2015)

La GRD es un ciclo que para ser eficaz, debe cruzar horizontalmente todos los procesos y actividades humanas y por lo cual, según Lavell (2007), debe además abarcar ciertos puntos que se encuentran estrechamente relacionados con el entendimiento de los procesos y actores sociales que contribuyen de manera directa o indirecta en la construcción del riesgo.

Son una gran variedad de sectores los involucrados en la GRD, por lo que, las decisiones dependen de las visiones que mantengan los y las participantes de la sociedad civil, actores privados y públicos entre los cuales es necesario crear y fortalecer la relación, con el objetivo de negociar el apoyo mutuo entre ellos y entre sus intereses sociales y territoriales. Por lo tanto, la GRD debe ser transversal a los procesos y actividades humanas, lo cual puede ser un desafío alcanzar debido a la existencia de políticas públicas precarias y falta de incentivos para lograr una concientización tal que facilite su desarrollo, en donde el Estado ya no solo genere medidas pos desastre debido a la ubicación de actividades productivas en zonas de riesgo, sino que se realicen acciones para lograr medidas ex ante que disminuyan las consecuencias potenciales (Orellana, 2015).

Para lograr una gobernanza efectiva dentro de la GRD, es necesario impulsar y crear formas institucionales y de organización que le den consistencia y pertinencia al diálogo entre los actores que desarrollen actividades en los distintos niveles territoriales. Es necesario definir responsabilidades, delimitar funciones y roles de los distintos participantes sociales organizados (Lavell, 2007). Es preciso mencionar que frente a esta situación, se debe tomar en consideración el contexto actual en el que algunos países sufren de inconsistencias, vacíos y contradicciones en sus instrumentos, así también entre sus autoridades, lo cual afecta también sus niveles de gestión: la existencia de intereses contrapuestos pueden dificultar el desarrollo de relaciones favorables en un contexto de GRD (Orellana, 2015). Según Cardona (2008, p.6), "en materia de riesgo público o colectivo, no solo los problemas, sino también las responsabilidades de la toma de decisiones son compartidas [...] Para resolver efectivamente esto se requiere de un proceso continuo de descubrimiento de elementos comunes entre diferentes grupos", y no solo desde el sector público, el cual constantemente se ve presionado por los ciudadanos, ya que lo perciben como el único responsable de la seguridad pública y bienestar común.

Es importante, por tanto, estimular convocatorias dirigidas tanto a fuerzas gubernamentales como no gubernamentales. En este sentido, parece sabio el incorporar los valores y preferencias de aquellos que son afectados por las decisiones en torno al riesgo, ya que estos grupos e individuos son los que después experimentarán los resultados de las decisiones acerca del riesgo (Renn, 2015). En este sentido, es importante descentralizar la GRD con el objetivo de que las comunidades e inversiones locales se involucren y puedan participar de los distintos procesos, logrando finalmente resultados efectivos de intervención (Cardona, 2008 en Orellana, 2015). Así como indica Cardona (2008, p.7), "El modelo de descentralización que se utiliza para análisis y toma de decisiones es condición necesaria para la real participación de la comunidad y de las autoridades locales [...] La autonomía de las comunidades locales y de sus propias autoridades debe ser una estrategia explícita para lograr resultados efectivos de intervención", siendo la gobernanza el medio para poder concretarlo. 
Por último, es importante recalcar que la base de todo proceso de gobernanza es la comunicación efectiva entre los actores, ya que en el diálogo puede existir retroalimentación de conocimiento entre ellos, además de permitir transparentar los intereses y valores, para poder incorporarlos y/o tomarlos en consideración en el proceso. Los mismos actores participantes poseen un rol clave al comunicar y divulgar las actividades y acuerdos que emanen desde el diálogo y acuerdos transversales hacia el resto de la sociedad (Renn, 2015).

\section{METODOLOGÍA}

Para el análisis de la planificación territorial en las comunas de San Bernardo y Calera de Tango se utilizaron como fuentes los instrumentos dispuestos que rigen a cada una de las comunas, además se realizaron levantamientos de uso de suelo a través del trabajo de campo para conocer el uso actual y concreto del espacio comunal. Finalmente, para identificar las gestiones municipales asociadas a la configuración del territorio, se realizaron entrevistas de tipo semi-estructuradas a funcionarios/as de los municipios en estudio.

Para comprender el contexto normativo en el que se encuentran insertas las comunas estudiadas, se identificaron todas aquellas leyes, políticas, planes y estrategias que tienen incidencia directa en el ordenamiento y planificación territorial en Chile, con el objetivo de analizar la normativa territorial respecto de su competencia en la GRD a diferentes escalas.

El análisis de estos instrumentos se realizó a través de una tabla comparativa en la cual se categorizó el contenido de cada uno dependiendo del tipo de concepto que según la teoría se está abordando y cómo lo está tratando cada instrumento, es decir, si es que este se ajusta a la teoría actual del riesgo de desastres o no. También esta tabla permitió realizar una comparación entre los documentos analizados: información con la que fue posible establecer patrones que se repiten en el abordaje del tema en cada una de las normativas territoriales.

Luego, este mismo análisis se realizó en los instrumentos de planificación y ordenamiento territorial de las comunas seleccionadas. En el caso de San Bernardo se analizó el Plan Regulador Metropolitano de Santiago (PRMS) año 1994 (SERVIU MINVU, 1994), el Plan Regulador Comunal (PRC) año 2006 y el Plan de Desarrollo Comunal (PLADECO) año 2011 (llustre Municipalidad de San Bernardo, 2006a, 2011). En el caso de Calera de Tango se realizó el análisis del PRMS año 1994, además se analizó el PLADECO año 2016 (Ilustre Municipalidad de Calera de Tango, 2016). Cabe destacar que en esta comuna no fue posible analizar su PRC ya que hasta la fecha no existe. El análisis de estos instrumentos respecto de su relación con la GRD y sus conceptos asociados permitió identificar la incorporación de mecanismos, conceptos, reglamento y zonificaciones asociadas a las áreas de riesgo en cada comuna.

Para complementar este análisis y con el objetivo de conocer la realidad actual y los procesos de generación de los instrumentos ya mencionados, se realizó una entrevista a un funcionario de la Asesoría Urbana de la Secretaría Comunal de Planificación (SECPLA) de la comuna de San Bernardo y en el caso de Calera de Tango, no fue posible entrevistar a algún/a funcionario/a de SECPLA, por lo que se realizó la entrevista al Encargado de Emergencia y a un funcionario del Departamento de Obras Públicas de la Municipalidad.

Luego de la búsqueda y análisis de estudios e informes de organismos técnicos, se da cuenta del escenario multi-amenaza en el que se encuentra cada comuna: esto, junto con la información de los usos de suelo establecidos por los planes reguladores, permitió generar un análisis espacial con el apoyo de dos tipos de cartografías por cada comuna, con el objetivo de evaluar el nivel de eficacia de la incorporación de la GRD en la normativa territorial local. Una de las cartografías tiene el objetivo de identificar los tipos de uso de suelo que se encuentran expuestos a amenazas y determinar si en estos existen o no zonas residenciales. Y la otra permitió contrastar los usos de suelo en la teoría de la normativa territorial con el real uso que se le da a los sectores cercanos a las áreas de riesgo en cada comuna.

Por último, en base a los resultados de los análisis anteriores y teniendo en consideración el contexto normativo en el que se insertan las comunas, se comparan los casos seleccionados, estableciendo factores críticos para definir determinados niveles de eficacia de la incorporación de la GRD. El análisis comparativo se realizó mediante tablas en base a los criterios (ver Tabla 1): 
Criterios para realizar análisis comparativo entre San Bernardo y Calera de Tango

\begin{tabular}{|c|c|c|}
\hline $\begin{array}{c}\text { roceso de generación del instrumento planificador y } \\
\text { organización municipal respecto a la GRD } \\
\text { desarrollo de cada instrumento, sus etapas, actores } \\
\text { involucrados, reuniones realizadas, enfoques, } \\
\text { objetivos, entre otros) }\end{array}$ & Uso de conceptos del riesgo & $\begin{array}{l}\text { Diálogo entre el contenido de los instrumentos y la } \\
\text { realidad comunal }\end{array}$ \\
\hline
\end{tabular}

Tabla 1. Criterios para realizar análisis comparativo entre San Bernardo y Calera de Tango Fuente: Autora, 2020

\section{RESULTADOS}

Incorporación de la GRD en instrumentos de ordenamiento y planificación territorial en diferentes escalas

El análisis de las leyes, políticas, planes y estrategias que se identificaron como relevantes en temas de ordenamiento y planificación urbana en Chile (ver Tabla 2) indica, en primera instancia, que existe un uso confuso de los conceptos asociados al riesgo, ya que no se mantienen dentro de lo que plantea la actual teoría respecto del tema en cuestión. La mayoría de los instrumentos se basan más bien en el estudio de las amenazas y exposición sin considerar las características de la vulnerabilidad, un tema confirmado también por Sandoval y Boano (2014).

\begin{tabular}{|c|c|c|c|c|}
\hline Escala Territorial & Tipo de instrumento & Instrumento & Carácter & $\begin{array}{c}\text { Actores y/o organismos } \\
\text { responsables }\end{array}$ \\
\hline \multirow{8}{*}{ Nacional } & Ley & $\begin{array}{l}\text { Ley General de Urbanismo y } \\
\text { Construcciones }\end{array}$ & Normativo & Ministerio de Vivienda y Urbanismo \\
\hline & Ley & $\begin{array}{c}\text { Ley Orgánica Constitucional } \\
\text { sobre Gobierno y Administración } \\
\text { Regional }\end{array}$ & Normativo & Ministerio del Interior, SUBDERE \\
\hline & Ley & $\begin{array}{c}\text { Ley Orgánica Constitucional de } \\
\text { Municipalidades }\end{array}$ & Normativo & SUBDERE \\
\hline & Ordenanza & $\begin{array}{l}\text { Ordenanza General de } \\
\text { Urbanismo y Construcciones }\end{array}$ & Normativo & Ministerio de Vivienda y Urbanismo \\
\hline & Política & $\begin{array}{l}\text { Política Nacional de Desarrollo } \\
\text { Urbano }\end{array}$ & Indicativo & Ministerio de Vivienda y Urbanismo \\
\hline & Política & $\begin{array}{c}\text { Política Nacional de Desarrollo } \\
\text { Rural }\end{array}$ & Indicativo & Ministerio de Agricultura, SUBDERE \\
\hline & Política & $\begin{array}{l}\text { Política Nacional de Desarrollo } \\
\text { de Localidades Aisladas }\end{array}$ & Indicativo & Ministerio del Interior- SUBDERE \\
\hline & Política & $\begin{array}{l}\text { Política Nacional de Uso de } \\
\text { Borde Costero }\end{array}$ & Indicativo & $\begin{array}{c}\text { Ministerio de Defensa, Subsecretaría } \\
\text { para las Fuerzas Armadas, ex } \\
\text { Subsecretaría Nacional de Marina }\end{array}$ \\
\hline \multirow{3}{*}{ Regional } & Estrategia & $\begin{array}{l}\text { Estrategia Regional de } \\
\text { Desarrollo }\end{array}$ & Indicativo & Gobierno Regional \\
\hline & Plan & Plan Regional de Gobierno & Indicativo & Gobierno Regional \\
\hline & Plan & $\begin{array}{c}\text { Plan Regional de Ordenamiento } \\
\text { Territorial }\end{array}$ & Indicativo & Gobierno Regional \\
\hline Intercomunal & Plan & $\begin{array}{l}\text { Plan Regulador Intercomunal o } \\
\text { Metropolitano }\end{array}$ & Normativo & Gobierno Regional \\
\hline \multirow{4}{*}{ Comunal } & Plan & Plan Regulador Comunal & Normativo & Municipalidad \\
\hline & Plan & Plan de Desarrollo Comunal & Indicativo & Municipalidad \\
\hline & Plan & Plan Seccional & Normativo & Municipalidad \\
\hline & Límite & Limite Urbano & Normativo & Municipalidad \\
\hline
\end{tabular}

Tabla 2. Instrumentos competentes en el ordenamiento y planificación territorial de Chile Fuente: Autora, 2020

Por otro lado, es posible identificar la gestión asociada a las áreas de riesgo en estudios, proyectos y propuestas que surgen desde los instrumentos, pero con un enfoque correctivo y reactivo. Si bien, existe un acercamiento a la prevención, preparación y mitigación, aún se trabaja 
sobre riesgos existentes y con una marcada preocupación por la emergencia más que en las otras etapas de la GRD. Además, la normativa de los instrumentos de planificación respecto de las áreas de riesgo, es muy general, no detalla actores claves, características de los estudios, períodos de tiempo, entre otros aspectos que al no ser explicitados en la normativa, provocan vacíos en ésta y problemas en su aplicación al hacer uso del territorio.

La antigüedad de los instrumentos analizados está directamente relacionada con la manera de tratar el riesgo de desastres. La Política Nacional de Desarrollo Urbano es el instrumento más reciente y el que aborda de manera más completa la temática. Los conceptos que utiliza concuerdan con la teoría actual del riesgo e incluso incorpora a la resiliencia como elemento importante en lo relacionado con la reducción de riesgos de desastres. La metodología del Plan Regional de Ordenamiento Territorial también tiene menos de 10 años, y es destacable dentro de este análisis por la visión intersectorial que posee y la profundización en el ámbito estudiado. La Ley General de Urbanismo y Construcciones es el instrumento más antiguo analizado y las falencias que posee en la utilización y comprensión de los conceptos reflejan la falta de consideración de la teoría del riesgo, lo cual es una debilidad importante, considerando que es el instrumento normativo más importante en la planificación territorial y contiene la reglamentación de los instrumentos de planificación territorial.

\section{Incorporación de la GRD en los instrumentos de ordenamiento y planificación locales y en la gestión municipal}

En San Bernardo, las 'áreas de riesgo natural' que se mencionan en los instrumentos que rigen a la comuna actualmente (PRMS y PRC), en realidad se refieren solo a áreas de amenaza pero no considera características de vulnerabilidad de la población por lo que no aborda de manera íntegra el riesgo, esto indica que se mantiene lo analizado anteriormente en los instrumentos de ordenamiento territorial en las distintas escalas. En el caso de Calera de Tango, ya que no tiene PRC, es el PRMS el instrumento que regula a la comuna actualmente. Este no considera áreas de riesgo en ella, por lo que tampoco considera elementos asociados a la GRD.

Luego de analizar la visión que tiene el PRMS en torno a ambas comunas, fue posible identificar que el instrumento en cuestión plantea distintas funcionalidades para cada una de ellas en base a sus características socio-territoriales y que esto puede afectar las gestiones municipales, sobre todo en lo relacionado con la reducción del riesgo de desastres. Por ejemplo en San Bernardo, algunos objetivos que plantea el PRC actual se han visto mermados por normativas que han surgido desde el PRMS, los cuales tendrán que ser asumidos de manera obligatoria en la actualización del instrumento comunal. Según lo indicado en la Memoria Explicativa del PRMS, Actualización áreas de extensión Urbana y Reconversión (PRMS100) año 2013, debido a las proyecciones de población al año 2030, el PRMS consideró que era necesario ampliar el área de extensión urbana en algunas comunas de la RM. San Bernardo es una de las que debió modificar su límite de extensión urbana, en específico hacia sectores que se encuentran expuestos a amenazas, como el sector norte, hacia el sector de Rinconada de Chena y hacia el sur, alrededor del río Maipo, esto debido a que pertenece al tercero de los cinco mercados geográficos de la región que compiten por recibir demanda de viviendas. Estas nuevas zonas de extensión urbana son denominadas 'Zonas Urbanizables Condicionadas' (ZUC), lo cual significa que la ocupación de estas debe responder al cumplimiento de una serie de reglamentos condicionantes que garantizan la factibilidad para ser urbanizadas.

La interacción entre ambos instrumentos se encuentra tensionada por los distintos fines que busca cada uno y las dificultades de esto las ha tenido que asumir el municipio, el cuál presenta limitaciones a la hora de poder generar una gestión de riesgo que no sea reactiva. En Calera de Tango, existe una serie de problemáticas de uso del territorio, las cuales han sido difíciles de poder contener por parte de la municipalidad sin el respaldo de un PRC, pero en que tampoco el PRMS ha considerado una evaluación de la planificación actual de la comuna, ya que aún se asume que es una comuna rural sin considerar que existe un rápido y desregulado crecimiento residencial. En efecto, la única modificación al PRMS de 1994 que se ha realizado respecto de Calera de Tango, data del año 2005 con la modificación 66 que incorpora y extiende las áreas de Interés Silvoagropecuario Mixto (ISAM).

Respecto de las gestiones a nivel municipal en cada comuna, la organización institucional que hoy en día lleva a cabo la municipalidad de San Bernardo para la toma de decisiones y elaboración de sus instrumentos contempla el apoyo y asesoría de comités formados por distintos departamentos y funcionarios, por lo que existe mayor comunicación y organización interna. El hecho de que la 
Asesoría Urbana no maneje información de riesgo de desastre es criticable desde el punto de vista del ordenamiento y planificación del territorio. Este debería generarse desde las características territoriales, contemplando sus amenazas, vulnerabilidad y exposición de la población y bienes. Con todo, la dinámica de trabajo que hoy en día lleva la municipalidad en la toma de decisiones y en la generación de instrumentos, hacen que sea un apoyo importante para la SECPLA y sus departamentos, ya que el Departamento de Emergencias, que es el que maneja esta información, puede aportar y orientar el proceso a través de estos comités de trabajo.

En Calera de Tango, la desconexión entre departamentos es notoria, lo cual genera dificultades en el manejo de información y finalmente paraliza la toma de decisiones. El manejo de información respecto al ordenamiento y planificación territorial en los departamentos entrevistados no es suficiente, además de no contar con cartografías con información comunal generada desde el mismo municipio: solo poseen la información del PRMS.

\section{Nivel de eficacia de la incorporación de la GRD en la normativa territorial local}

Como información base para analizar las comunas seleccionadas, se identificaron las amenazas tanto de origen natural como antrópico presentes en cada una. En el caso de San Bernardo, éstas son: inundaciones y anegamientos (cortes viales producto de las inundaciones) que ocurren en algunos sectores pavimentados y pasos bajo nivel, considerando siempre como un foco de tensión las riberas del río Maipo; incendios de vegetación en zona urbana mayoritariamente concentrados en el cerro Chena; y remociones en masa presentes en los cerros Chena y Lonquén. Por su parte, Calera de Tango se ve amenazado principalmente por: inundaciones y anegamientos; y remociones en masa en las zonas de los cerros Chena y Calera.

En San Bernardo y Calera de Tango los usos de suelo permitidos en sus instrumentos de planificación en las zonas amenazadas, en general, no involucran zonas residenciales. Sin embargo, estas albergan actividades económicas productivas, las cuales, de ser afectadas, impactarían de manera negativa en la recuperación de la población ya que son parte de sus medios de vida. Es el caso de Calera de Tango, en que los tipos de uso de suelo que mayoritariamente se verían afectados bajo las amenazas identificadas en esta investigación, corresponden a Áreas de Interés Agropecuario Exclusivo (ver Figura 2).

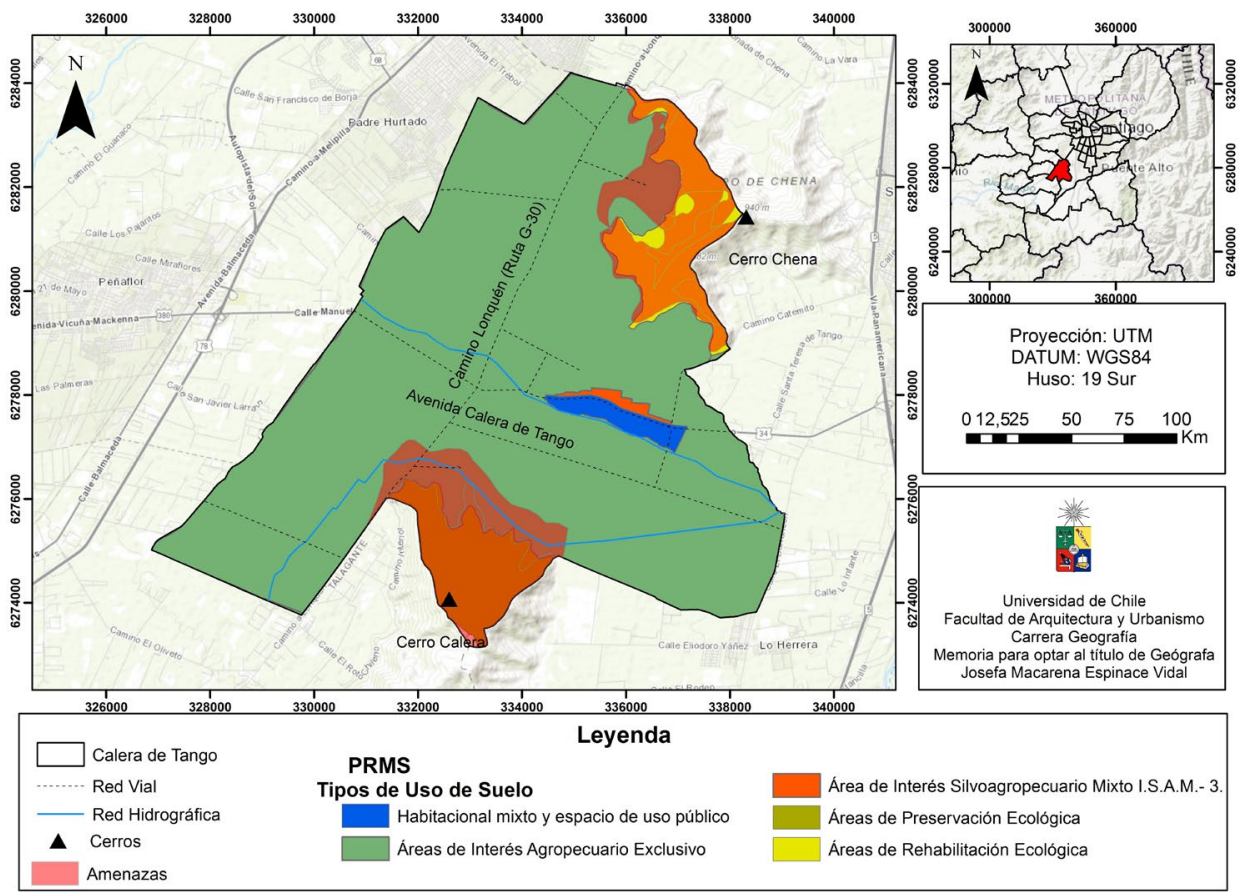

Figura 2. Zonas altamente amenazadas en la comuna de Calera de Tango, superpuestas a los usos de suelo según Plan Regulador Metrop litante amenazas en

Fuente: Autora, 2020. Las zonas amenazadas corresponden a elaboración propia en base al estudio Bases para un ordenamiento territorial ambientalmente sustentable para la RM (OTAS), II Etapa: Inventario ecológico y evaluación de territorio del Gobierno Regional de la RM del año 1998

Si bien el PRMS de 1994 no considera zonas de riesgo en Calera de Tango, no significa que no existan áreas altamente amenazadas por distintas características presentes en la comuna. Como muestra la Figura 3, que contiene los usos de suelo levantados en terreno, existen zonas 
amenazadas aledañas a los cerros Chena y Calera donde no se respeta la normativa que dice relación con los usos permitidos en el Área de Interés Agropecuario Exclusivo. Además, el uso de suelo es mayoritariamente de tipo extractivo en sectores de plantaciones y agroindustrias asociadas a ellas. Sin embargo, no es insignificante la cantidad de uso de tipo residencial, ya sean viviendas unifamiliares agrupadas 0 individuales.

En los alrededores del cerro Calera existen distintos tipos de usos (Figura 3). Además del extractivo y el residencial, es posible encontrar comercio mayorista y minorista, también servicios públicos, sociales y comunitarios. El uso de servicio público, que se encuentra al poniente del cerro, corresponde a una planta de recepción de reciclaje. Por otro lado, el uso de servicio social y comunitario corresponde a un centro de salud y a dos colegios. En el caso del centro de salud, este es considerado infraestructura crítica, ya que, según UNISDR (2009), estas son instalaciones que son esenciales para el funcionamiento de una sociedad o comunidad, tanto en la vida cotidiana como durante emergencias.

En este sentido, es posible determinar que de ocurrir un evento natural extremo asociado al cerro Calera, existe infraestructura crítica y residencial que sufriría consecuencias negativas, lo cual además implica que una cantidad importante de población que se vería afectada.
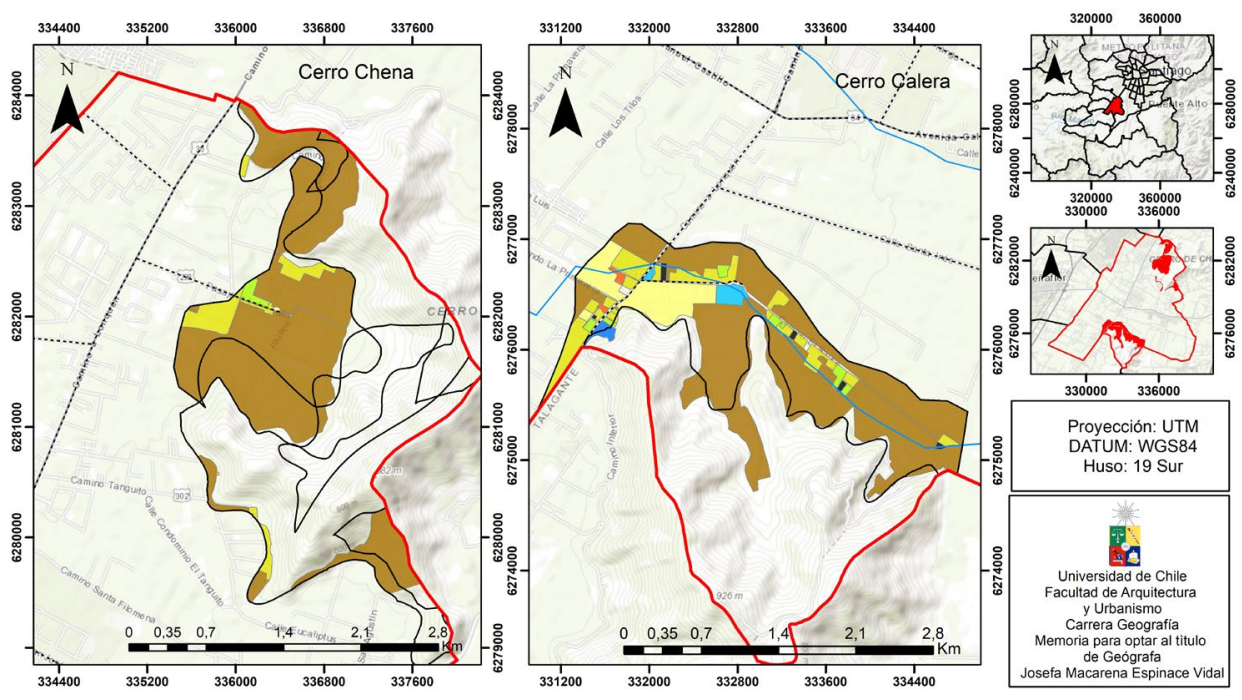

\begin{tabular}{|c|c|c|}
\hline \multicolumn{3}{|c|}{ Leyenda } \\
\hline & Levantamiento uso de suelo & \\
\hline$\square$ Calera de Tango & Comercio Mayorista y Prestación de Servicios Especiales (B2) & Sector de Servicios Sociales y Comunitarios (A2) \\
\hline ….... Red Vial & Comercio Minorista y Prestación de Servicios Profesionales (B1) & Sin uso \\
\hline —_ Red Hidrográfica & Industria Extractiva (C4) & Unifamiliar Agrupada (D2) \\
\hline$\square$ Amenazas Físicas & Sector de Servicios Públicos (A3) & Unifamiliar Puntual (D1) \\
\hline
\end{tabular}

Figura 3. Uso de Suelo actual en zonas altamente amenazadas en la comuna de Calera de Tango

Fuente: Autora, 2020, basado en levantamiento de información realizado el día 12 de agosto de 2018. Las zonas amenazadas corresponden a elaboración propia en base al Estudio Bases para un ordenamiento territorial ambientalmente sustentable para la RM (OTAS), II Etapa: Inventario ecológico y evaluación de territorio del Gobierno Regional de la RM del año 1998.

En el caso de San Bernardo, su PRC permite un uso mixto en una zona que se encuentra altamente amenazada por dinámicas del cerro Negro, la cual puede albergar desde residencias hasta colegios (ver Figura 4). Por otro lado, también se encontrarían expuestas las Áreas de Interés Agropecuario Exclusivo, lo cual afectaría los medios de vida de la población ante la ocurrencia de un evento natural extremo. En este sentido, es posible identificar que la normativa no consideró las características físico-naturales del territorio al momento de su elaboración, situación que más que reducir el riesgo, lo aumenta. Es importante mencionar también que en ninguna de las comunas se consideran zonas de 'amortiguación' (buffer zones) entre las áreas bajo amenaza y los usos colindantes, sean estos residenciales o industriales.

Si bien existe regulación del suelo en los instrumentos planificadores de la comuna de San Bernardo y ésta se cumple en su mayoría, no es así respecto de las áreas de riesgo que en ellos se identifican. Los sectores altamente amenazados corresponden a los cerros presentes en la comuna y los alrededores del río Maipo.

Según el levantamiento de uso de suelo realizado en terreno, existen residencias (unifamiliar 
agrupada e individual o puntual) en algunas áreas expuestas a amenazas, en particular alrededor del cerro Negro y en áreas cercanas al río Maipo. Respecto de las que se encuentran alrededor del cerro, si bien no son grandes extensiones de zonas habitadas, algunas de éstas se encuentran en áreas altamente expuestas (ver Figura 5). Por otro lado, según la zonificación del PRC, también están expuestas zonas de uso mixto, por lo cual es posible encontrar también zonas residenciales. En consecuencia, los usos de suelo permiten áreas residenciales y de educación (infraestructura crítica) en áreas amenazadas: el colegio ubicado en el sector se encuentra dentro de la normativa, respetando los instrumentos planificadores.
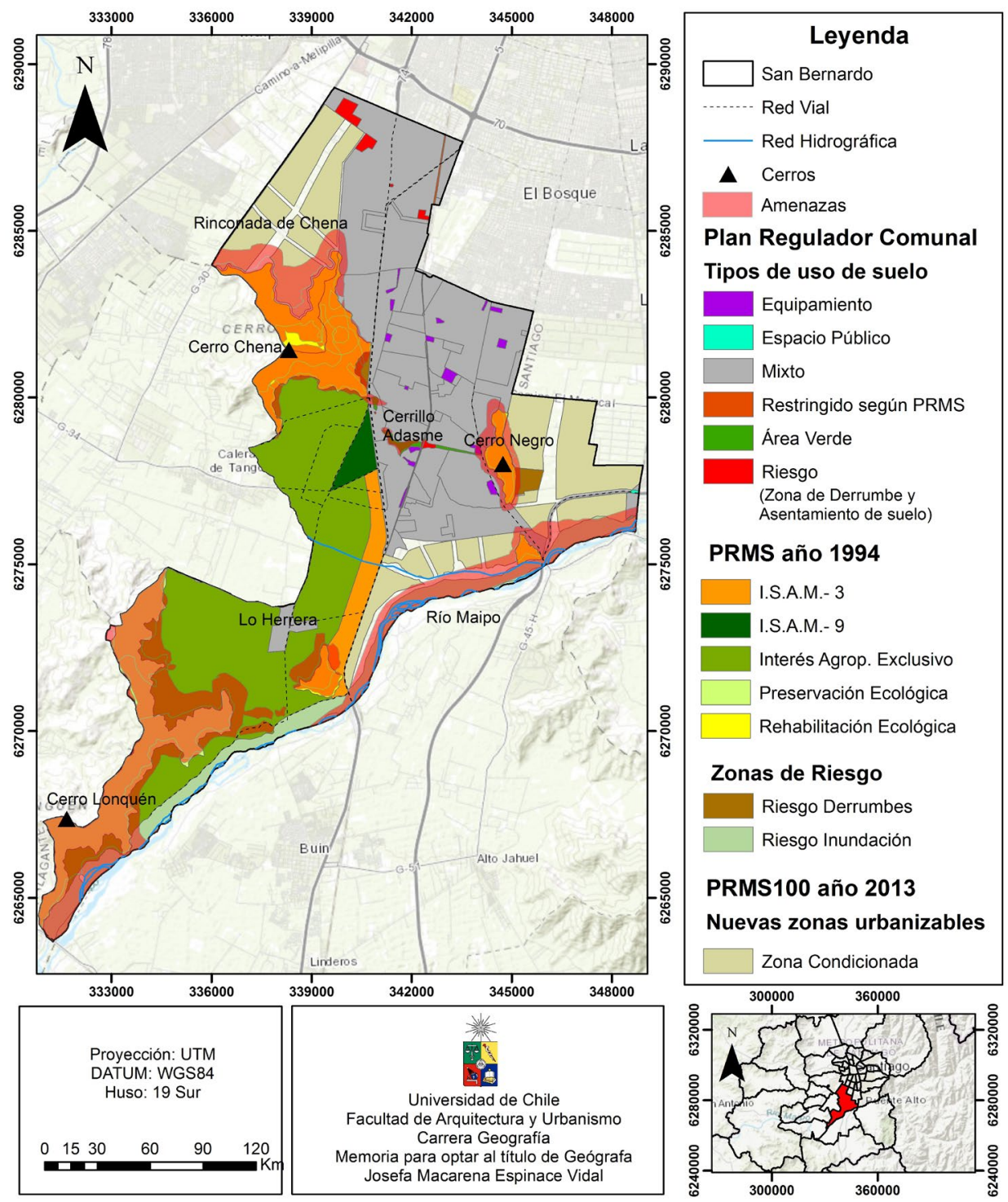

Figura 4. Zonas amenazadas en la comuna de San Bernardo, superpuestas a los usos de suelo según Plan Regulador Comunal y Plan Regulador Metropolitano de Santiago

Fuente: Autora, 2020. En el caso del PRC y PRMS, en base a información geoespacial del SEREMI MINVU año 2015, a través de la página web de Infraestructura de Datos Geoespaciales del Ministerio de Bienes Nacionales. Las zonas amenazadas corresponden a elaboración propia en base al Estudio Bases para un ordenamiento territorial ambientalmente sustentable para la RM (OTAS), II Etapa: Inventario ecológico y evaluación de territorio del Gobierno Regional de la RM del año 1998, y estudios y bases de datos de CONAF (2018) ${ }^{1}$.

También hay casos alarmantes. Alrededor del cerro Chena existen escasas zonas habitadas y es posible encontrar un colegio en el límite urbano-rural de la comuna. Si se considera que está dentro del área urbana, el colegio no cumple con la normativa, ya que esa es una zona industrial exclusiva cuyos usos permitidos no se encuentra la de educación -excepto por establecimientos de formación técnico-profesional (llustre Municipalidad de San Bernardo, 2006b). Sorprendentemente, en caso de pertenecer al área rural, tampoco cumple la normativa, ya que en las Zonas Urbanas Condicionadas el equipamiento permitido no contempla educación (SEREMI MINVU, 2018).

Es posible determinar que aun cuando la normativa planteada responda a las características y necesidades de la población y tenga entre sus objetivos la reducción del riesgo, si ésta no es respetada y fiscalizada, los esfuerzos desde la planificación serán en vano, dejando en manos de

1. Información obtenida a través de Ley de Transparencia (https://www. consejotransparencia.cl) 
los municipios la tarea de reducir los riesgos. De esta forma, Calera de Tango y San Bernardo están actualmente incrementado la exposición de los asentamientos humanos que se están ubicando en áreas que sufrirían consecuencias importantes ante un evento asociado a las amenazas presentes en cada comuna.
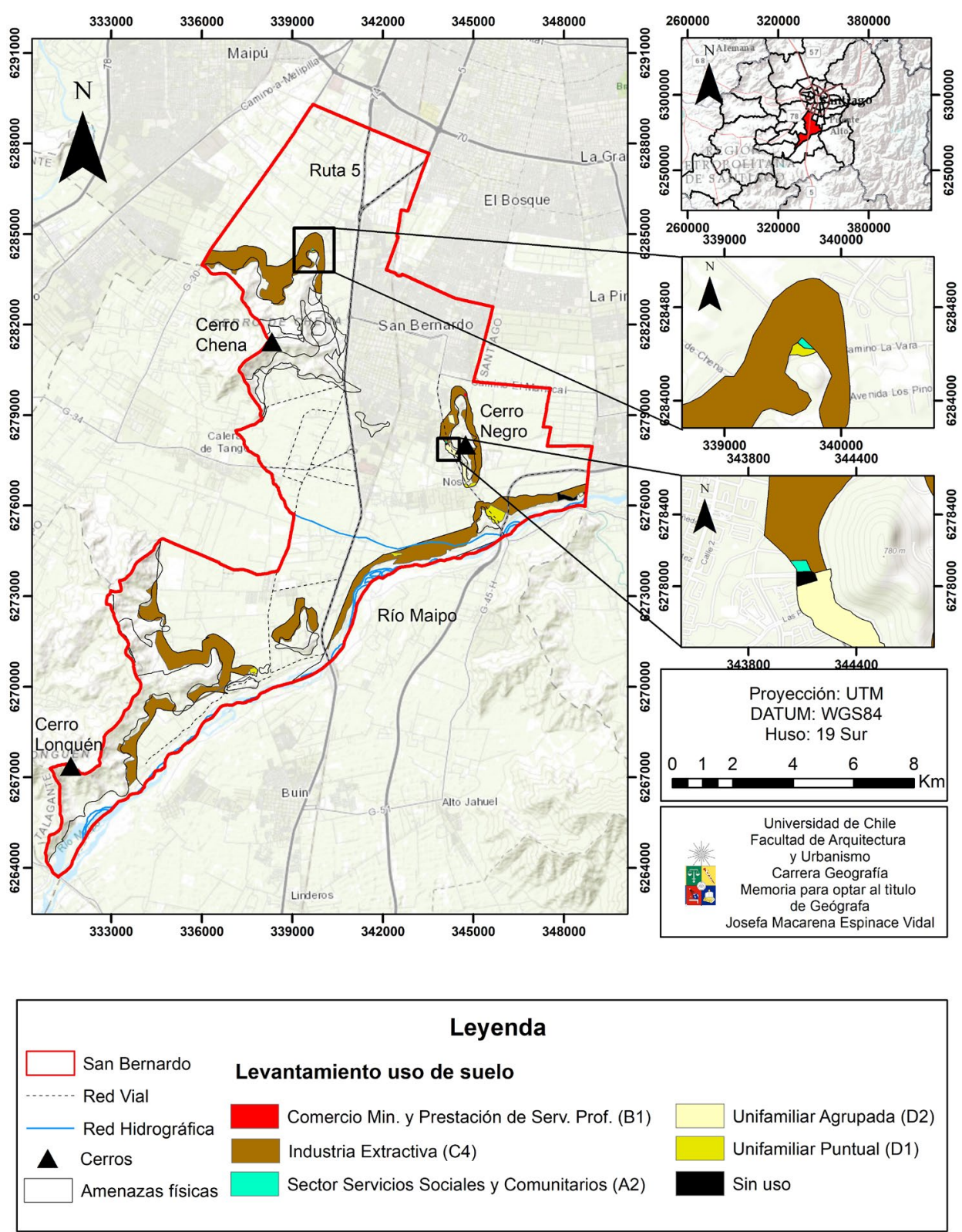

Figura 5. Uso de suelo actual en zonas altamente amenazadas en la comuna de San Bernardo

Fuente: Autora, 2020, basado en levantamiento de información realizado el día 12 de agosto de 2018. Las zonas amenazadas corresponden a elaboración propia en base al estudio Bases para un ordenamiento territorial ambientalmente sustentable para la RM (OTAS), II Etapa: Inventario ecológico y evaluación de territorio del Gobierno Regional de la RM del año 1998, y en el caso de los incendios según estudios y bases de datos de CONAF.

\section{DISCUSIONES}

Luego de revisar el estado del arte en torno a la incorporación de la GRD en los instrumentos de planificación y ordenamiento territorial en Chile, es posible determinar que por diferentes razones ésta aún es incipiente. En primera instancia, se evidencia un uso confuso de los conceptos, los cuales se encuentran distantes de la teoría actual del riesgo. Este último concepto aún no se entiende como un proceso asociado a una serie de factores y procesos socio-territoriales: en efecto, la mayoría de los instrumentos más bien se basan en estudios de amenazas aceptando solo la existencia de fenómenos físico-naturales presentes en el territorio como causas de los desastres, sin vincularlos con la relevante componente social del riesgo, la vulnerabilidad y los procesos de desarrollo. Esto puede deberse a que existe una débil consciencia de que el riesgo es una construcción social, en donde la acumulación histórica de intervenciones humanas en el espacio han dado lugar a un incremento de los eventos dañinos (García Acosta, 2005), lo que también ha 
conllevado a un crecimiento importante de los niveles de vulnerabilidad.

Por otro lado, la antigüedad de los instrumentos analizados está directamente relacionada con la manera de tratar el riesgo de desastres. La Ley General de Urbanismo y Construcciones es el instrumento más antiguo analizado y las falencias que posee en la utilización de los conceptos reflejan su desfase en la comprensión de la teoría del riesgo dominate actual, lo cual es preocupante considerando que es el instrumento normativo más importante en la planificación territorial del país y contiene la reglamentación de los IPTs. En este sentido, es realmente importante considerar la actualización de estos, ya que las dinámicas de la sociedad, y en particular de aquellas urbanas, crea constantemente nuevos escenarios posibles de desastre, generando nuevas o modificadas amenazas y vulnerabilidades, desafiando el estado del conocimiento actual del territorio y a la vez, las medidas de gestión que ya existen (Mansilla, 2000). Por lo tanto, mientras el uso del territorio sea dinámico, su planificación también debe serlo.

El estado general de los instrumentos de ordenamiento y planificación territorial respecto de la incorporación de la GRD en ellos, se ve reflejado en el trabajo que se desarrolla en los niveles locales. La gestión del riesgo en sus instrumentos de planificación también es correctiva y reactiva, mientras que la confusión de conceptos se mantiene. Esto puede deberse a que aún no se concibe la relación de la gestión del riesgo con el ordenamiento territorial, lo cual debe ser subsanado considerando que la ocupación desregulada del territorio provoca una serie de amenazas, que en conjunto con los focos de vulnerabilidad existentes en la población, han incrementado los niveles y zonas de riesgos (Arenas et al., 2010). Los departamentos de planificación municipal trabajan sin conexión con los departamentos de gestión del riesgo o de emergencia, no relacionando aún sus áreas de trabajo en la toma de decisiones. De hecho, es difícil que se involucre el área de emergencias actual en gestiones de largo plazo porque como su nombre lo indica, son departamentos que se enfocan en la reacción al corto plazo. La desconexión entre las distintas funciones que existen en los municipios limita la organización y la calidad de la gestión que se pueda llevar a cabo en el territorio.

Esta situación podría mejorar si se tuviera en consideración que la prevención del riesgo se encuentra directamente relacionada con las restricciones de uso del espacio, tarea que el ordenamiento territorial es capaz de ejercer a través de los instrumentos de planificación dispuestos (Gómez Orea, 1994, en Ayala-Carcedo, 2001).

Por otro lado, la gestión municipal de ambas comunas también se encuentra limitada porque debe absorber las decisiones que se han definido desde los macro niveles y que no siempre se han mantenido en concordancia con la visión que tiene el municipio de la comuna. El PRMS enfoca la planificación territorial mayoritariamente en torno a las necesidades que surgen desde el centro urbano de la región, decisiones que no necesariamente van en beneficio de todas las comunas involucradas. Así, San Bernardo ha ido incorporando a su territorio las demandas habitacionales que surgen desde la región producto del explosivo crecimiento urbano que se ha registrado en el último tiempo, lo cual ha traído como consecuencia la generación de nuevos riesgos, situación que se potencia por la presión desde el sector inmobiliario para construir en sectores peri urbanos (Arenas et al., 2010). Con todo, al presentar dificultades para satisfacer la demanda de servicios e infraestructura para estas nuevas urbanizaciones, la comuna parece sumirse en un deterioro progresivo de condiciones sociales y medioambientales (Sánchez, 2010).

En Calera de Tango, si bien no se ha definido desde el PRMS como un polo de atracción residencial, el instrumento de regulación no da cuenta de las dinámicas que se están desarrollando en la comuna, que sumado a una débil gestión al respecto, está generando la construcción de nuevas áreas de riesgos. Al considerar a Calera de Tango como una comuna de carácter principalmente rural con un área urbana de muy baja superficie, no se generan mayores esfuerzos por planificar el uso del territorio. En este sentido, es posible identificar algunas consecuencias del sesgo urbano que posee la instrumentación territorial en el país: los centros urbanos han adquirido una alta centralización y concentración de actividades (Sánchez, 2010), también la población se concentra en estos lugares, por lo que se pueden asociar a zonas que necesitan un trato particular enfocando todos los mecanismos de gestión en ellas. No obstante, las áreas rurales también se encuentran habitadas y debido a la falta de planificación en estas, son asentamientos humanos cada vez más expuestos a las amenazas presentes en su territorio. En efecto, hoy en día es un número no menor de residencias, además de dos colegios y un servicio de salud, los que se encuentran expuestos a las amenazas de origen natural asociadas a la dinámica de los cerros de Calera de Tango. Los actores de la gobernanza del riesgo, en espacial el Estado por su rol principal, deben hacerse cargo de la situación a través de los instrumentos que poseen a su favor. Si Calera de Tango contara con un PRC, aunque no sería la solución definitiva a la problemática existente, podría ser un aliciente para 
comenzar a regular y gestionar su territorio con una visión local que el PRMS no le puede entregar.

La gama de instituciones de los distintos niveles territoriales que interviene en el espacio es amplia, y aunque posean mayor o menor nivel de intervención, complejizan el panorama para el desarrollo de una política integrada, planes coherentes e instrumentos efectivos (Bustos, 1998). Esta realidad se evidencia en ambas comunas, donde la tensión existente entre los instrumentos de planificación, los cuales finalmente responden a las visiones que emanan desde los macro niveles territoriales, dificulta de manera importante la gestión del riesgo de desastres en el nivel local.

Pese a que una gran variedad de factores y procesos intervienen en la construcción del riesgo desde la escala nacional y regional, el nivel comunal es la primera escala territorial en percibir y enfrentar las situaciones de desastre, por lo que son los niveles que pueden identificar de mejor manera los mecanismos de prevención, preparación y mitigación que necesitan para reducir el riesgo. Las decisiones en los macro niveles no deberían tomarse sin considerar los contextos, visiones y gestiones locales, pero actualmente sucede que las decisiones en torno a las problemáticas asociadas al desarrollo y el ordenamiento territorial se han enfrentado desde una perspectiva parcial y centralizada (Bustos, 1998). El centro de estos procesos es la acción colectiva, voluntaria y descentralizada, consciente de la necesidad de reducir el riesgo, basada en la comunicación, retroalimentación y auto-organización (Cardona, 2008), considerando entonces como base a la gobernanza para poder llevar a cabo procesos efectivos de reducción del riesgo de desastres.

La zonificación que se genera desde los instrumentos de planificación territorial tampoco puede definirse sin considerar las características del espacio a ordenar. El establecimiento de criterios es la base para generar dicho orden, lo cual la vuelve una actividad proactiva en lugar de reactiva (Arenas et al., 2010), al considerarse variables de importancia al largo plazo y las consecuencias que estas podrían provocar en la población (UNISDR, 2009). En San Bernardo y Calera de Tango, los usos de suelo permitidos en las zonas amenazadas, en general, son coherentes con la condición del territorio, ya que no involucran zonas residenciales. Sin embargo, son zonas que albergan actividades económicas productivas, las cuales de ser afectadas, impactarían de manera negativa en la recuperación de la población ya que son parte de sus medios de vida.

Es importante mencionar que en ninguna de las dos comunas se consideran 'zonas de amortiguación' entre las áreas bajo amenaza y los usos colindantes, sean estos residenciales o industriales. Este tipo de situaciones se podrían evitar con el mejoramiento de la coordinación entre las instituciones responsables que participan tanto de la GRD como del ordenamiento territorial, para que se generen planes coherentes que logren proteger el uso adecuado del suelo en función de los contextos de cada comuna (Castro et al., 2013).

El Estado de Chile es responsable de entregar las condiciones mínimas, y de manera equitativa a las diferentes comunas, para que sean capaces de generar sus instrumentos de planificación y ordenamiento territorial, y todo tipo de herramienta que sea necesaria para gestionar el uso del espacio y reducir el riesgo de desastres a través de una gestión efectiva y de largo plazo.

\section{CONCLUSIONES}

Los instrumentos que abordan de mejor manera la gestión del riesgo de desastre en el país, son todos indicativos (no vinculantes), y aquéllos que son normativos (vinculantes, en el sentido legal) se encuentran desactualizados en materia de ordenamiento territorial. En este sentido, es importante cuestionar el tipo de mecanismo que se está utilizando para generar los cambios y el orden esperado en el espacio. Al actualizar los instrumentos normativos y potenciar el uso y seguimiento de aquellos indicativos, se podrían direccionar de mejor manera los esfuerzos para reducir el riesgo de desastres.

El análisis comparativo entre ambas comunas ha permitido identificar diferencias y similitudes entre las comunas de San Bernardo y Calera de Tango. Uno de los elementos que comparten es que ambas comunas muestran cierta confusión en la utilización de conceptos asociados al riesgo de desastres, lo cual se ve reflejado en sus instrumentos de ordenamiento y planificación territorial. Esta problemática puede ser subsanada con la socialización e internalización en la gestión institucional, del glosario de conceptos elaborada por la Plataforma Nacional para la Reducción del Riesgo de Desastres (PNRRD) liderada por la Oficina Nacional de Emergencia (ONEMI).

También hay algunas observaciones. Si bien San Bernardo posee características en su gestión 
municipal y en sus instrumentos de planificación que le favorecen para gestionar el riesgo, como la zonificación, estas características siguen siendo insuficientes o desaprovechadas para incorporar una mejor gestión de los riesgos en los instrumentos de ordenamiento y planificación territorial.

Por otro lado, la elaboración del Plan Regulador Metropolitano de Santiago debería sustentarse en la gobernanza para ser capaz de acoger y atender a las distintas necesidades que surgen desde las comunas que regula. La flexibilidad del instrumento ante el cambio y/o alteración de los planes reguladores comunales permite que éste sea capaz de mantener el equilibrio entre todos los territorios que tenga a su alcance. Los procesos que ocurren a escala local van determinando y alterando las dinámicas en niveles mayores, por lo que no considerarlos dentro de la planificación intercomunal, genera tensiones y desequilibrios que pueden traer como consecuencia la generación de nuevos riesgos por los distintos usos que se le da a espacios comunes, como por ejemplo el cerro Chena en el caso de San Bernardo y Calera de Tango.

Con todo, este trabajo no ha podido identificar claramente la capacidad de articular y equilibrar las diferentes visiones de ocupación del espacio en los instrumentos que entrega la legislación vigente, en pos de reducir el riesgo de desastres y potenciar ciudades sustentables.

Finalmente, se espera que la investigación realizada aporte una conversación crítica y constructiva hacia la gestión institucional futura, respecto de la visión y decisiones que se tomen para hacer uso del territorio, considerando la gestión del riesgo de desastres entre sus principales mecanismos de trabajo.

\section{REFERENCIAS}

Arenas, F., Lagos, M. \& Hidalgo, R. (2010). Los riesgos naturales en la planificación territorial. Santiago de Chile: Instituto de Geografía, Pontificia Universidad Católica de Chile.

Ayala-Carcedo, F. (2001). La Ordenación del territorio en la prevención de catástrofes naturales y tecnológicas. Bases para un procedimiento técnico-administrativo de evaluación de riesgos para la población. Boletín de la A.G.E. №30, 2000, 37-49.

Barrenechea-Riveros, F. (2016). Comunas prioritarias para la gestión del riesgo de desastres: un aporte a la toma de decisiones. Revista Geográfica de América Central, 2(57).17-42. https://doi.org/10.15359/ rgac. $57-2.1$

Bustos, N. (1998). El ordenamiento y planificación territorial en Chile: elementos para su discusión. Revista de Geografía Norte Grande, 25, 49-53.

Cardona, O. (2008). Medición de la Gestión del Riesgo en América Latina. Revista Internacional de Sostenibilidad, Tecnología y Humanismo, 3, 1-20.

Castro C., De Souza V. \& Vásquez K. (2013). Los drivers del riesgo en la ciudad de La Serena, Chile: exposición, vulnerabilidad subjetiva e institucional. En Arteaga A., C. \& Tapia Z., R., Vulnerabilidades y desastres socionaturales. Experiencias recientes en Chile (pp.79-94). Santiago de Chile: Editorial Universitaria. http://repositorio.uchile.cl/handle/2250/136617

Corporación Nacional Forestal CONAF. (2018). Base de datos incendios forestales en la comuna de San Bernardo año 2012 - 2018. Santiago de Chile: CONAF.

García Acosta, V. (2005). El riesgo como construcción social y la construcción social de riesgos. Desacatos, Revista de Ciencias Sociales, 19, 11-24. http://ref.scielo.org/n37d 7x

Gómez-Orea, D. (1994). La Ordenación del Territorio. Una aproximación desde el Medio Físico. Madrid: ITGE-Editora Agrícola.

Gómez-Orea, D. (2007). Marco Conceptual de la Ordenación Territorial. En Ordenación Territorial (pp. 29-72). Madrid, Barcelona, \& Ciudad de México: Mundi-Prensa Libros.

Gross, P. (1998). Ordenamiento Territorial: El manejo de los espacios rurales. Revista Latinoamericana de Estudios Urbano Regionales EURE, 24(73), 116-118. http://dx.doi.org/10.4067/ $\underline{\text { So250-71611998007300006 }}$

Ilustre Municipalidad de Calera de Tango. (2016). Plan de Desarrollo Comunal Calera de Tango. Calera de Tango, Chile: SECPLA.

Ilustre Municipalidad de San Bernardo (2006a). Ordenanza Local Plan Regulador comuna de San Bernardo y localidad de Lo Herrera. San Bernardo, Chile: Municipalidad de San Bernardo. Recuperado de: http://www.sanbernardo.cl/contenidos/transparencia/plan.regulador.html 
Ilustre Municipalidad de San Bernardo. (2006b). Memoria Explicativa Plan Regulador comuna de San Bernardo y localidad de Lo Herrera. San Bernardo, Chile: Municipalidad de San Bernardo. Recuperado de: http://www.sanbernardo.cl/contenidos/transparencia/plan.regulador.html

Ilustre Municipalidad de San Bernardo. (2011). Tomo I Caracterización y Diagnóstico Comunal. San Bernardo, Chile: Municipalidad de San Bernardo. Recuperado de: www.sanbernardo.cl/contenidos/ transparencia/plan.desarrollo.comunal.html

Instituto Nacional de Estadísticas INE. (2018) Censo 2017. Santiago de Chile: INE. Recuperado de http:// www.cens02017.cl

Lavell, A. (2007). Apuntes para un reflexión institucional en países de la Subregión Andina sobre el enfoque de la Gestión del Riesgo. Lima: Comunidad Andina. Recuperado de: http://www. comunidadandina.org/predecan/doc/ri/docAllan2.pdf

Mansilla, E. (200o). Riesgo y Ciudad. San Jose, Costa Rica: Red de Estudios sociales en Prevención de Desastres en América Latina, La RED.

Oficina de las Naciones Unidas para la Reducción del Riesgo de Desastres UNISDR. (2009). Terminología sobre Reducción del Riesgo de Desastres. Ginebra: UNISDR.

Oficina Nacional de Emergencia del Ministerio del Interior ONEMI. (2017). Identificación Factores Subyacentes del Riesgo de Desastres. Instructivo Equipo Comunal. Santiago de Chile: ONEMI.

Orellana, J. (2015). Actividades económicas y su rol en la construcción social del riesgo. El caso de la comuna de Caldera, región de Atacama [Tesis Pregrado]. Santiago de Chile: Universidad de Chile.

Renn, O. (2015). Stakeholder and Public: Involvement in Risk Governance. International Journal of Disaster Risk Science, 6(1), 8-20. https://doi.org/10.1007/s13753-015-0037-6

Sánchez, R. (2010). La debilidad de la gestión del riesgo en los centros urbanos. El caso del área Metropolitana de Santiago de Chile. Revista de Geografía Norte Grande, 47, 5-26. http://dx.doi. org/10.4067/So718-34022010000300001

Sandoval, V. \& Boano, C. (2014). The Progression of Vulnerability: A Multi-Scalar Perspective on Disasters. The Case of Chaitén in Chile. In Calandra, L. M., Forino, G. \& Porru, A. (Eds.), Multiple Geographical Perspectives on Hazards and Disasters (pp.29-40). Rome: VALMAR. https://doi. org/10.13140/RG.2.1.3081.4885

Secretaría Regional Ministerial Metropolitana del Ministerio de Vivienda y Urbanismo SEREMI MINVU. (1994). Memoria Explicativa del Plan Regulador Metropolitano de Santiago. Santiago de Chile: MINVU.

Secretaría Regional Ministerial Metropolitana del Ministerio de Vivienda y Urbanismo SEREMI MINVU. (2005). Memoria Explicativa MPRMS - 66. Incorpora Nueva ISAM 9, Suprime ISAM 2 en San Bernardo, Extiende ISAM 3 en San Bernardo y Calera de Tango. Santiago de Chile: MINVU.

Secretaría Regional Ministerial Metropolitana del Ministerio de Vivienda y Urbanismo, SEREMI MINVU. (2013). Memoria Explicativa. Modificación Plan Regulador Metropolitano de Santiago MPRMS 10o. Actualización Áreas extensión urbana y reconversión. Santiago de Chile: MINVU.

Secretaría Regional Ministerial Metropolitana del Ministerio de Vivienda y Urbanismo, SEREMI MINVU. (2015a). Zonificación Planes Reguladores Comunales Región Metropolitana. En Infraestructura de Datos Geoespaciales. Santiago de Chile: IDE Chile.

Secretaría Regional Ministerial Metropolitana del Ministerio de Vivienda y Urbanismo, SEREMI MINVU (2015b). Zonificación Plan Regulador Metropolitano de Santiago (PRMS). En Infraestructura de Datos Geoespaciales. Santiago de Chile: IDE Chile.

Secretaría Regional Ministerial Metropolitana del Ministerio de Vivienda y Urbanismo, SEREMI MINVU. (2018). Texto refundido y sistematizado. Ordenanza Plan Regulador Metropolitano de Santiago, PRMS. Santiago de Chile: MINVU. Recuperado de: http://metropolitana.minvu.cl/pag-m/ documentacion-vigente-prms/

Universidad de Chile. (1997). Bases para un ordenamiento territorial ambientalmente sustentable para la Región Metropolitana (OTAS). En II Etapa: Inventario ecológico y evaluación de territorio (pp. 117-134). Santiago de Chile: Facultad de Ciencias Físicas y Matemáticas, Departamento de Ingeniería Civil, Universidad de Chile. Recuperado de: http://www.sectra.gob.cl/biblioteca/detalle1. asp? $\mathrm{mfn}=2832$ 